\title{
Doing Social Research on Online Communities: The Benefits of Netnography
}

\author{
By Felice Addeo*, Angela Delli Paoli ${ }^{\dagger}$,Maria Esposito ${ }^{*}$ \& \\ Maria Ylenia Bolcato ${ }^{\S}$
}

\begin{abstract}
In social science research, Netnography has become a widely accepted research method. It has been used to tackle a wide variety of topics from culture to identity, social relationships and civic empowerment. Netnography can be broadly defined as a qualitative research approach that adapts the traditional ethnographic techniques to the study of the "net", which is the online communities, practices and cultures formed through computer-mediated communications. Both Ethnography and Netnography are naturalistic and unobtrusive approaches, interested in studying social practices in their everyday context (Kozinets 2010). They are both multi-method, methodologically flexible and adaptive, not confining themselves to following specific procedures, but rather remaining open to issues arising from the field (Varis 2016). However, Netnography differs from Ethnography under some crucial points. Entering the online culture diverges from face-to-face entrée in terms of accessibility and research design. From a data collection perspective, Netnography is far less time consuming; however, it requires a new set of skills due to the specificities of computer-mediated communication and its dramatically increased field site accessibility, which requires choices about field sites and decisions about types of data to gather and analyse. Moreover, it is far less intrusive than traditional Ethnography as it allows for researcher invisibility: the cyberspace makes it possible for researchers to be unseen from people observed. This allows documenting the explicit language of informants without the risk of obtrusiveness and disturbance. This paper presents the methodological specificities of Netnography focusing on its context of application, the definition of the method, the research design: from the objectives and research questions' setting, to sites' selection and cultural entrée, from the type of data to be collected, to the way to classify, analyse and represent them. The paper will also discuss some examples of netnographic studies in social sciences.
\end{abstract}

Keywords: Netnography, Online Communities, Qualitative methods, Social research methods.

\section{Introduction}

The growing number of internet-based studies, along with constant developments in computer-mediated communication, offer a new field for social research, while simultaneously presenting many methodological challenges. The paper focuses on a specific research method which can be listed as a qualitative

\footnotetext{
*Associate Professor, University of Salerno, Italy.

${ }^{\dagger}$ Research Fellow, University of Salerno, Italy.

Teaching Assistant, University of Salerno, Italy.

$\S$ Teaching Assistant, University of Salerno, Italy.
} 
research approach alternatively named as Netnography (Kozinets 2002, 2010, 2015); Cyber Ethnography (Morton 2001), Ethnography of Virtual Spaces (Burrel 2009), Internet ethnography (Boyd 2008), Ethnography on the Internet (Beaulieu 2004), Internet related ethnography (Postill and Pink 2012); Digital Ethnography (Murthy 2008), Webnography (Puri 2007). Among these alternative labels, according to us, the one that best captures the nature of this method is Netnography as it clearly suggests the idea of a qualitative research approach that adapts the traditional ethnographic techniques to the study of the "net" that is the online communities, practices and cultures formed through computer-mediated communications.

The term, while obviously recalling the traditional ethnography, suggests, at the same time, the idea that doing online ethnographic research is something different from its offline parallel.

Ethnography is the study of social interactions, behaviours and perceptions of communities in their own time and space and in their own everyday lives (Burawoy et al. 1991), in order to produce detailed, rich, holistic and situated accounts and understanding of the cultures, perspectives, practices and social actions of the people in these settings, i.e. thick descriptions (Geertz 1973). "Thick description" is an expression used to characterize the process of paying attention to contextual detail in observing and interpreting social meaning when conducting during a qualitative research. A thick description of a social event or action takes into account not only the immediate behaviours in which people are engaged but also the contextual and experiential understandings of those behaviours that render the event or action meaningful (see the Sage Research Methods Dictionary).

Both Ethnography and Netnography are naturalistic and unobtrusive approaches, interested in studying social practices in their everyday context (Kozinets 2010). They are both built on the combination of different research methods, are methodologically flexible and adaptive, not confining themselves to following specific procedures, but rather remaining open to issues arising from the field (Varis 2016). However, Netnography differs from ethnography under some crucial points. From a data collection perspective, Netnography is far less time consuming; however, it requires a new set of skills due to the specificities of computer-mediated communication and its dramatically increased field site accessibility, which requires choices about field sites and decisions about types of data to gather and analyse. Moreover, it is far less intrusive than traditional ethnography as it allows for researcher invisibility: the cyberspace makes it possible for researchers to be invisible to people observed. This allows documenting the explicit language of informants without the risk of obtrusiveness and disturbance. This paper presents the methodological specificities of Netnography focusing on its context of application, the definition of the method, the research design: from the objectives and research questions' setting, to sites' selection and cultural entree, from the type of data to be collected, to the way to classify, analyse and represent them. The paper will also discuss some examples of netnographic studies in social sciences, and finally conclusions are drawn. 


\section{The Context of Netnography: Online Social Spaces}

Computer mediated communication (CMC) is being incorporated into every aspects of daily life: everyday people worldwide are using blogs, social networks, chat rooms, personal worldwide pages and other online channels to express their identity, share information, ideas, and values, build knowledge, common practices and relationships (Riffle et al. 2019, Hallett and Barber 2013, Kozinets, 2010, 2002, Garcia et al. 2009, Mann and Stewart 2000).

Social life has been deeply penetrated by the Internet (Beneito-Montagut 2011); the use of online spaces to build communities and social relationships with people independently from their geographical location is widely spreading.

These virtual spaces take the form of small-scale communities without established parameters but held together through shared emotions, styles of life, new moral beliefs, and senses of injustice and consumption practices (Cova 1997). Following the definition of Rheingold (1993: 5) - who developed the expression "virtual communities"- they can be referred as social aggregations that emerge from the net when a large number of persons - moved by sufficient human feelings- carry on argumentized and long discussions about a domain of interest developing webs of personal relationships in cyberspace.

They are emotionally based, and they can connect heterogeneous people having different socio-cultural backgrounds, but sharing at the same time interests, passions and feelings. Virtual communities assume the form of communities of practice (Wenger 1998) when members, linked by common interests and passions, become content producers and practitioners developing an extended repertory of resources in order to share information, create ideas, find common solutions, build knowledge, make innovations, and so on (Lave and Wenger 1991). These communities are based on distinctive systems of meanings that are either exclusively or mainly manifested and negotiated online.

The participation in virtual communities is not restricted to just one community: people engage in different communities basing on their personal interests. The majority of the people, during their lives, usually takes part in several communities through different media, technologies and platforms, depending on the passions and the interests they perceive as predominant in a specific period of their existence. Netnography is a non-media-centric approach: the media are not the focus of research, netnographic research is not interested in media characteristics and use (Pink et al. 2016) but instead in the cultures, experiences, activities and relationships developed through different media (social networking groups, blog, communities, etc.), in one word online "worlds of meanings" (Kozinets 2015). Within this perspective, online social spaces can cross the boundaries of one specific community and platform and can have a collectively distributed nature. Consider for example the case of social media where a group of people may coordinate through using the same hashtag in order to share interests, opinions, emotions and so on. In this case a technical strategy (the use of the same hashtag) pairs up with a discursive strategy (Caliandro 2018).

Considering the need for social and community belonging behind online spaces' participation, Maffesoli (1996) rejects the assumption of the triumph of 
individualism to connote postmodernism. Instead, postmodernism seems to be characterized by a contrary movement toward the end of individualism expressed by the anxious search for social and community links. The same perspective is expressed by Cova (1997: 300-301); to him, post-modernity creates new social compositions: "the individual who finally managed to liberate him/her from archaic or modern social links is embarking on a reverse movement to recompose his/her social universe on the basis of an emotional free choice".

Participation in online spaces is a relevant part of daily life and relationships developed within online communities can, and often do, cross the communities' boundaries and affect other aspects of people social life. This is due to recent technological developments which increase the scope and range of online communities and the forms and time of participation such as the web 2.0 which widened the opportunities for user-generated content, the emergence of an "internet of things" and of ubiquitous mobile devices which make it possible to be always connected (Costello et al. 2017). Many studies have demonstrated that online communities contribute to change notions of the self, have identity implications, become systems of social support, institutionalize power and support activism (Olaniran 2008, Campbell 2006, Gossett and Kilker 2006, Madge and O'Connor 2006, Carter 2005, Williams and Copes 2005).

Thus, the distinction between online and offline life and the dichotomy realvirtual is more and more blurred losing its usefulness. In other words, virtual reality is not something distinct from other aspects of human actions and experience, but rather a part of it (Costello et al. 2017, Hallett and Barber 2014, Beneito-Montagut, 2011, Kozinets 2010, Garcia et al. 2009). Digital spaces are embedded in our culture so to change social practices (Hallet and Barber 2014). As a result of these changes, most sociologists think that, to really understand current society, it is necessary to follow people's social activities, including Internet and other forms of CMC. This implies an adjustment to epistemological and methodological stances for doing social research and an adaption of traditional social research methods to the specificities of online interactions.

\section{Many Names, Same Approach?}

Historically, social research on online communication articulates into two stages.

A first phase is dominated by experimental research with the aim of testing the effects of communication technologies without considering contextual factors (Androutsopoulos 2006: 4).

The second stage is characterized by a "[...] growing application of naturalistic approaches to online phenomena and the subsequent claiming of the Internet as a cultural context", with ethnographic research increasingly applied (Hine 2005: 7).

However, the ethnographic methods to study online social interactions are still undefined and in flux. The uncertainty concerns even the different labels that social researchers use to describe their studies of online communities and cultures. 
Some researchers (Garcia et al. 2009, Kanayama 2003, Maclaran and Catterall 2002) simply call their online studies "ethnography", intending an inclusive and complex approach that does not substantially change whether it is used for studying an offline or online phenomenon.

Hine (2005) talks about "virtual ethnography", considering it as a partial and limited approach because it is focused just on the online aspects of the social life and does not consider the entire social experience.

Exploring the literature, it is possible to come across many other labels, such as "cyber ethnography" (Robinson and Shulz 2009, Morton 2001), "ethnography of virtual spaces" (Burrel 2009), "Internet ethnography" (Boyd 2008), "ethnography on the Internet" (Nelson and Otnes 2005, Beaulieu 2004), "Internet related ethnography" (Postill and Pink 2012), "digital ethnography" (Hjorth et al. 2017,Varis 2016, Murthy 2008), "webnography" (Horster and Gottschalk 2012, Puri 2007, Evans et al. 2001), online ethnography (Tuncalp and Lé 20014).

Many other scholars (La Rocca et al. 2014, Beaven and Laws 2010, Maulana and Eckhardt 2007, Fuller et al. 2006, Nelson and Otnes 2005), following the Kozinets proposal (1998), use the term "Netnography" to describe their studies about online communities.

All these labels refer to some kind of online data and to ethnography as a research approach (Varis 2016).

This paper adopts the term "Netnography" basing on several reasons:

- the term, based on a combination of the words "Internet" and "ethnography", immediately and clearly suggests the idea of a qualitative research approach that adapts the traditional ethnographic techniques to the study of online communities and practices;

- the term, while obviously recalling the traditional ethnography, suggests, at the same time, the idea that doing ethnographic research on online communities is something different from its offline parallel. As it will be shown in the following pages, Netnography distinguish itself from ethnography under many points of view;

- by now, the term is established and accepted by social researchers and frequently recurs in the academic literature about the study of online communities (unlike other labels that sporadically occur in isolated contribution about the topic).

In agreement with many other scholars (Beneito-Montagut 2011, Kozinets 2010), this work does not favour the expression "virtual ethnography", because it assumes that the virtual reality is something different and separated from the real and suggests a dichotomy real-virtual that, considering the spread of CMC in individual and social everyday life, does not exist anymore.

\section{Netnography as a Research Method}

Netnography as research approach has been developed by Robert Kozinets (1998) in the area of marketing and consumer research. Over the last decade, the 
approach has found application in a lot of social sciences areas, from sociology and anthropology to cultural and media studies.

Netnography can be defined as a qualitative research approach that transposes and adapts the traditional, in-person ethnographic research techniques to the study of the online cultures and communities formed through computer-mediated communications (Kozinets 2002: 2).

Through the use of not intrusive observation techniques, Netnography allows the researchers to study social interactions online, immersing themselves in the virtual environment in which these interactions are performed. It allows studying particular online cultures in their manifestation.

The specific purpose of Netnography is therefore represented by the study of online social spaces as previously defined. It is not limited to the analysis of the individualistic and sporadic action of posting messages on the internet, but it explores continued and repetitive interactions performed trough computer channels and technologies through the analysis of online communication. Online communication can take many forms, including text, but also audio information, visual information and audio-visual information. Depending on the research topics and aims, communities under analysis can take the forms of blogs, forums, social networks, chats, mailing lists, play spaces, virtual worlds, wikis, etc.

The origins of Netnography can certainly be traced in its offline parallel, traditional ethnography.

Ethnography belongs to the sphere of qualitative approaches within which it occupies a prominent role. It is a social research method aimed to study cultural knowledge, patterns of social interaction, particular society, drawing on a wide range of source of information. The main source of information is the ethnographer, overt or covert, participation in target people's daily life for an extended period, collecting observational data on the issues of analysis. The goal of any ethnography is to study people, culture and society in their own time and space and in their own everyday lives (Burawoy et al. 1991: 2), in order to produce detailed and situated accounts, i.e. thick descriptions (Geertz 1973). "Thick description" is an expression used to characterize the process of paying attention to contextual detail in observing and interpreting social meaning when conducting qualitative research. A thick description of a social event or action takes into account not only the immediate behaviours in which people are engaged but also the contextual and experiential understandings of those behaviours that render the event or action meaningful (Sage Research Methods Dictionary).

The main advantage is its capacity to depict the perspectives of actors, the richness and complexity of social life challenging the misleading preconceptions that social scientists often bring to research. Ethnography is able to produce an authentic understanding of a culture based on concepts that emerge during the study, instead of being imposed a priori from the researcher hypotheses (Hine 2005). This is the reason why ethnography has been recognized as a particularly suitable approach to familiarize the unknown, i.e. to describe backgrounds of social life that are difficult to access otherwise. At the same time, it is appropriate to deconstruct what is an already known, revealing new and surprising features of well-known social contexts (Marzano 2004). In doing so, researchers are required 
to be accurate, sensitive and reflective toward their subjects/objects of analysis and the context in which these subjects are acting and performing (Beneito-Montagut 2011).

Hammersley and Atkinson (2007) identify the main features of the ethnographic research:

1. the approach requires the immersion of the researcher in the everyday life of a social group for an extended period of time, in order to directly observe the group members and often participate in the group activities;

2. the ethnographic research takes place "in the field": distinct from other techniques as experimental, in ethnographic research people are studied in their everyday context and life, rather than under conditions artificially created by the researcher;

3. ethnographers prefer an inductive approach in which the assumptions directly emerge from the fieldwork rather than a deductive approach, in which assumptions are derived from the theories and then tested in the empirical world;

4. information derives from a variety of different sources, like documents, artefacts and, above all, participant observation and informal or formal interviews with members;

5. data collection is unstructured under two main points of view. First, it is not based on a fixed and rigid research design defined at the beginning of the research. Second, the categories for interpreting the phenomenon under study are not established a priori by the researcher, but they progressively emerge during the research process itself;

6. the research is usually focused on one or few cases because the main research goal is not to generalize, but reach an in-depth understanding of the phenomenon under study, gaining a type of particularized knowledge that is grounded in a specific context;

7. the analysis of data involves a hermeneutic interpretation of the members' meanings, actions and practices. The analysis is mostly based on verbal descriptions and explanations. Quantification and statistical analysis play a very subordinate role;

8. the entire ethnographic process is characterized by a strong unpredictability, as it represents a unique and unrepeatable encounter between a researcher and a social context in a specific historical time.

Most of these features apply also to Netnography. It is possible to identify a lot of common elements between ethnography and Netnography. They are both naturalistic approaches, interested in studying social experiences and practices in their everyday context (Kozinets 2010); they are both based on adaptation or bricolage, being always built on the combination of different research techniques and methods. Both approaches are methodologically flexible and adaptive, not confining themselves to following specific procedures, but rather remaining open to issues arising from the field (Varis 2016).

Despite these common elements, there are also elements of distinction between the two research methods. Differently from ethnography, Nethnography is easier and far less consuming: information can be downloaded from the internet without having to be recorded and transcribed as in the traditional ethnography, 
the researcher does not have to travel anywhere for data collection. Moreover, it allows research invisibility making it possible for researchers to be invisible to people they are observing. In online settings, in fact, it is possible to lurk at the sites of interest, without disclosing the researcher's presence. Traditional ethnographies, instead, are unavoidably intrusive, as they involve a participant observation of the researcher and require at least the minimal participation of being there (Puri 2007, Evans et al. 2001). Another advantage of Netnography is the possibility of archiving material: as historical data are archived on most sites; it is possible to study trend over time. The permanence of online material also makes it possible to revisit sites at different times and do rigorous checks of consistency (Puri 2007). At last, Netnography also offers the opportunity to study real time trends: online conversations are always up to date, which makes it possible to study trends in real time, as soon as they form (see Table 1).

Table 1. Ethnography vs. Netnography: Similarities and Differences

\begin{tabular}{|l|c|c|}
\hline & Ethnography & Netnography \\
\hline Multi-method & $\mathrm{X}$ & $\mathrm{X}$ \\
\hline Context-specific & $\mathrm{X}$ & $\mathrm{X}$ \\
\hline Travel required & $\mathrm{X}$ & $\mathrm{X}$ \\
\hline Manual data recording & & $\mathrm{X}$ \\
\hline Automatic data recording & & $\mathrm{X}$ \\
\hline Unobstrusiveness & & $\mathrm{X}$ \\
\hline Studying trends over time & & \\
\hline $\begin{array}{l}\text { Studying trends in Real } \\
\text { time }\end{array}$ & & \\
\hline
\end{tabular}

Source: Authors own elaboration based on Kozinets' works.

\section{Types of (N)ethnographic Research}

Depending on the nature of the phenomenon researcher is trying to investigate, Netnography can be used as the only methods or supplemented by other research methods. In this regard, Kozinets (2010) introduces an important distinction between online communities and communities online, that can be very useful to understand if and when Netnography can be used as a stand-alone approach or if it should be used as a part of a larger study including other face-toface research techniques.

Doing research on online communities means studying phenomena specifically related to online communities and online culture. In studies of this type, the online interaction/communication/identity is central and an online research approach could legitimately be the only one. In studies of this type, the online setting is part of the definition of the phenomenon under study: community members interact and communicate mainly via Computer Mediated Communication (CMC) so that the researcher can decide to focus their attention only on the online setting.

On the other hand, the expression communities online refers to phenomena whose social existence extends beyond the Internet and online interaction, even if 
the virtual plays an important role within the phenomena themselves.

It is clear that this dichotomy represents just a schematic attempt of categorizing phenomena, maybe over simplistic, as the difference between online communities and communities online should be better read as a continuum. However, this classification could be useful for researchers and allows deriving a general guideline: research on online communities should tend to have a primarily netnographic focus; instead, in research on communities online, Netnography could play a supporting or a secondary role within the research process (Kozinets 2010).

A similar classification has been proposed by Garcia et al. (2009), who distinguish between social phenomena which exist solely or primarily online, i.e. groups whose members interact only or mainly via CMC, and multimodal social phenomena, that are performed through both CMC and face-to-face contacts. For the solely or primarily online phenomena, it is legitimate for ethnographers to study the community life and practices just by examining the member online behaviour. For the multimodal phenomena, instead, it is necessary to define the research setting combining online and offline components.

It comes from this also the distinction between Pure Netnography and Blended Netnography. A Pure Netnography is a research that is conducted only using data generated from online interactions. On the opposite, a Pure Ethnography is a research conducted totally using data collected face to face, with no data gathered from online interactions. A Blended Netnography, instead, would be a combination of the both approaches, including data collected face to face as well as by online interactions (Kozinets 2010) (see Table 2).

At the beginning of every research, the nethnographers must question themselves if they are studying a solely or primarily online community/ phenomenon. If it is, they can decide to adopt Netnography as a stand-alone method. In this case, a pure Netnography would be appropriate, exhaustive and complete. On the opposite, if the researcher is studying a more complex phenomenon, then limiting it to a netnographic study would be partial and incomplete.

According to many authors (Beneito-Montagut 2011, Garcia et al. 2009), the application of a pure ethnography will increasingly reduce through the time. As the distinction between online and offline worlds will be always less fuzzy, ethnographers must alter their research techniques, to the point that all ethnographies of contemporary society should include and integrate $\mathrm{CMC}$ in their definition of the research field.

Table 2. Typology of (N)ethnographic Studies

\begin{tabular}{|c|c|c|c|}
\hline & \multicolumn{2}{|c|}{ Data Collection } \\
\hline & & Face to face & Online \\
\hline \multirow[t]{2}{*}{$\begin{array}{l}\text { Type of } \\
\text { Interaction }\end{array}$} & Face to face & Pure Ethnography & Blended Ethnography \\
\hline & Online & Blended Netnography & Pure Netnography \\
\hline
\end{tabular}

Source: Adapted from Kozinets, 2010. 


\section{Netnography: Research Design}

In social research in general but in qualitative research in particular, research design is not rigid but malleable and the phases can be greatly modified or reimagined to suit specific projects. The evolution of a netnographic research, even more than for other qualitative research methods, cannot be predetermined, anticipating all its possible issued and planning strategies and solutions to face them (Hammersleyand Atkinson 2007, Marzano 2006). However, this unpredictability does not eliminate the need for a pre-fieldwork preparation and does not mean that the researcher's behaviour in the field can be totally casual and offhand, based on the mere attempt to accommodate events. As for other research approaches, research design is crucial also to Netnography, even if it is an open and flexible design in which a reflexive process operates throughout every stage of the research itself.

In general, we can say that the design of a netnographic research involves different phases. These phases do not need to be interpreted as sequential and linear stages as feedback, reversibility and inversion of phases is frequent in concrete research.

We can identify 6 different phases:

- defining research questions;

- selecting the research field;

- gaining access to the field;

- collecting information;

- analysing data;

- writing a research report.

\section{Defining Research Questions}

Research should always begin with some problems or set of issues, starting from what in 1922 Malinowski (2) referred to as "foreshadowed problems" (2002). One of the most important tasks in the early stages of the research is to turn the foreshadowed problems into a set of defined questions (Hammersley and Atkinson 2007). Authors disagree about the reference to theory at this stage. Some (Creswell 2008, Glaser and Strauss 1967) reject referring to the existing literature and theory in order to not contaminate the emergence of new issues. Kozinets (2010), instead, suggests that researchers should always have as much knowledge as possible about what other people have done in the same research area, trying to connect their works with a large frame of references and build bridges with the related literature in the same area. This depends also on the object of the study: if exploratory then the researcher may collect the information first and then try to make sense of it referring to theory; if descriptive then the researcher would refer to theory first to obtain information on the particular features of an issues and then collect the information. Consequently, we can imagine two main types of netnoghraphic studies: exploratory netnographies, i.e. studies in which the researcher tries to look for patterns in the data and come up with a model within which to view this data, and descriptive netnographies which are theoretically- 
driven starting from a gap in the relevant literature and dissatisfaction for the absence of detailed knowledge about a specific phenomenon.

Independently from the type of netnographic research, as for traditional ethnography, much of the research effort is concerned with formulating and reformulating the research questions in ways that make it more prolific and/or more suitable to investigation.

Although there are no hard-and-fact rules, Creswell (2008) suggests some useful guidelines for writing research questions during a qualitative research process:

- asking one or two central questions followed by a limited number of sub questions;

- beginning the research questions with the words what and how that suggest an open-ended research design;

- employing non-directional language, exploratory verbs, for example "discover", "understand", "explore", "describe", "seek to understand", "build meaning";

- using open-ended questions.

Once defined the (more or less structured) research questions, the researcher may use cognitive tools that facilitate the identification of significant dimensions in order to answer the research questions. In particular, conceptual maps could be very useful. They are taxonomic diagrams where an abstract concept - too general and abstract to be assessed - is progressively connected to more specific concepts. In this way, conceptual maps contribute to convert concepts into indicators for not directly measurable concepts (Marradi 2007: 203-204).

\section{Selecting the Case}

Apart from defining the research questions, it is necessary to find the best communities to start the field work (Silverman 2010, Kozinets 2010, Hammersley and Atkinson 2007). There is no a fixed order between the definition of the research question and the community selection. Sometimes the setting selection comes first, for example when the researcher participates directly to an interesting online community. In this case, foreshadowed problems are identified ex post and derive from the nature of the setting itself.

In other cases, the setting may be selected on the basis of foreshadowed problems. Even in this case, the nature of the setting may still affect the development of the research questions. This happens because, as underlined before, in netnographic research the development of research problems is rarely completed before fieldwork begins; indeed, data collection often plays a key role in that process of problems development.

After identifying the main research questions, the researchers are rarely in a position to directly specify the precise nature of the best setting for their research. At the beginning, they can try to identify the types of locations that would be most appropriate for investigating the research problems. The best strategy is using a variety of keywords, progressively refining and specifying the initial research keywords, until the research results are not satisfactory. At this stage, substantial 
differences emerge between traditional and online ethnography. Researchers need to explore a different number of options before finding the best community for their research purposes. Looking for a research field online is very different from doing it offline. While traditional ethnographers had to travel a lot in order to study a particular culture, the netnographers can perform the same work using a good search engine and entering terms related to the research area and focus (Kozinets 2010).

As for traditional ethnography, netnography uses a type of sampling that is known as theoretical sampling (Glaser and Strauss 1967). In this type of sampling, the purpose is to collect data from places, people and events that will maximise opportunities to develop many categories and properties of categories as possible, and to facilitate the emergence of relations among the categories themselves.

In other words, cases are selected according to their capability to give useful insights, rather than being aimed at producing findings that are representative of a population or at testing hypotheses.

Transposing the concept of theoretical sampling in online settings could be practical translated in following specific criteria and preferring online communities that are (Kozinets 2010):

- relevant, i.e. related to the issues under analysis, to the specific research focus and questions;

- active, as they do not need to be past communities but need to be present communities with ongoing, recent and regular communications;

- interactive, as they need to be relational spaces with active communications between participants;

- substantial, as they need to be spaces of discussion on specific issues with a critical mass of communicators;

- heterogeneous, with a number of different participants;

- data rich, with detailed and descriptive data.

\section{Gaining Access to the Field}

Before gaining access to the selected community, it is necessary to become familiar with the community itself, its rules, rituals, values, language, norms, practices, organization and members. In this explorative step, the researcher can try to find out, for example, who are the most active participants, who are the leaders, which are the most popular topics, what type of specialized language, rituals, activities and values are shared by the community members (Kozinets 2010). During this step, the researchers can really assess the feasibility of their research aim and objectives, defined only theoretically until that moment.

After having familiarized with the community, researchers need to gain access to it. Gaining access to a community represents a strategic moment on which the success or failure of the research can depend. As for ethnographic studies, netnographies are exposed to the risk of failure because of the lack of access to the field or the difficulty for researcher to establish a relationship with members and/or gain their trust (Hammersley and Atkinson 2007). Depending on the type of community, strategies to gain access may be different. Online 
environments can be mainly classified into two categories:

- open environments, whose contents are free and accessible to anyone;

- closed groups, where the contents are accessible upon user's registration which is, in turn, subject to the approval of one or more administrators.

Based on the type of community, the research aims and the features of the research field, as in ethnographic research, there are two basic ways of gaining access to the search site:

- covert access or lurking: the researcher invisibly observes the community without informing people about the research and the researcher's presence within the group with related ethical implications;

- overt access: the researcher informs community members of the research and asks for their consent, often through the gatekeepers (Silverman, 2010: 5), focal actors who act as gates and intermediaries for entering the community.

In the case of overt access which can be equated to participant observation in ethnography, there are different degree of participation in the community: researchers can decide to interact in a very limited way, just informing members about the research purposes and asking them some clarifying questions. They can also decide to interact with members as full participants, giving their contribution to the shared knowledge and practices. Thus, the participation of researchers in the community can be perceived as a continuum. On one hand, there is the observational role of the researcher, highlighting the possibility of observing without establishing any social contact with the community members. On the other, the participative role can be taken to the extreme and assume the form of an auto-Netnography, where the aim of the research becomes autobiographical reflections about the researcher own experience about his/her online community membership (see Figure 1).

Between these opposite stages there are a lot of different strategies of participation, at various levels and with different intensity, in the community under study (Kozinets 2010, Morton 2001):

- reading message in real time to following links;

- rating, commenting, replying to other members' messages;

- using chat lines and other synchronous forms of CMC for 'interviewing' by posting one question at a time and being able to immediately respond to the other;

- using mail for a different form of "interviewing" in which one or more questions can be sent at a time, and the subjects can respond in their own time;

- getting involved in the community activities and practices, becoming an expert within the community itself. 
Figure 1. The Level of Netnographer's Participation in the Online Community

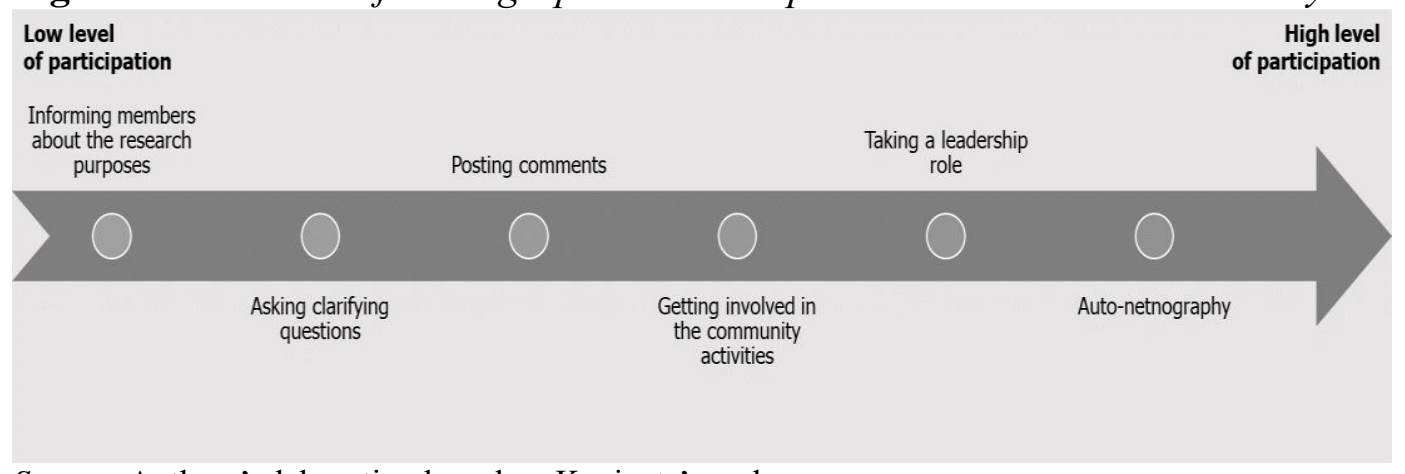

Source: Authors' elaboration based on Kozinets' works.

The choice between covert and overt access to the community is controversial. Covert access may have benefits in terms of obtrusiveness but has ethical and privacy implications. Instead, overt access is ethical correct but obtrusive. Some scholars (Kozinets 2010, Hine 2005) support overt access for ethical reasons: a researcher should always disclose his/her presence and research intentions to online community. Others (Hewer and Browmlie 2007, Beaven and Laws 2007, Langer and Beckmann 2005) believe that informing communities' members on the researcher's identity would compromise the main advantage of the approach, i.e. its unobtrusiveness. Arguments around the invisibility issue range a lot.

On the one hand, there are scholars (Beneito-Montagut 2011, Hine 2005, Bell 2001, Heath et al. 1999) who suggest that lurking is not ethnographic observation in the traditional sense and hence not "proper" ethnography. Lurking is not considered research in its real sense of collaboration, it is considered rather as a one-way process of appropriation, as an unethical practice which does not provide any deep understanding of the community, but only a superficial description due to the absence of dialogue with respondents (Bell 2001, Heath et al. 1999, Garcia et al. 2009).

On the other hand, there are scholars (Beaven and Laws 2007, Hewer and Browmlie 2007, Puri 2007; Langer and Beckmann 2005) that idealize the possibility of lurking thinking that it should be the norm for netnographic research. Lurking, in fact, offer a unique opportunity for collecting "natural" data, as the members are not aware of their informant status and do not modify their behaviour due to the researcher's presence. As Paccagnella (1997) points out, in social sciences, as well as in other fields, the phenomena under study are modified by the act of observing them. Even in the case of qualitative methods as the participant observation, the presence of a researcher in the field can cause problems. The author recognises that, while the possibility of performing observation without informing the people under study clearly poses new ethical issues, at the same time, it reduces the danger of bias and distorting data caused by the presence of the researcher.

Between these two extremes positions (never lurking or always lurking), there are authors who believe that lurking may be justifiable and legitimate in certain circumstances. Some authors (Garcia et al. 2008) underline that lurking could be 
acceptable at some conditions, especially if that is the way how members routinely participate and experience the community. In other words, researcher could experience the online sites in the same way that participant routinely experience it, gaining access to a field by displaying competence of the norms of the group and adapting their behaviour on the basis of them. Others (Maclaran and Catterall 2002) justify the choice of non-participant observation when the researcher thinks that the presence of an outsider within the community would be undesirable and unwelcome. Others (Langer and Beckmann 2005) consider a covert netnography as a particularly suitable approach to investigate sensitive topics, for which it is not advisable to apply traditional research methods as a questionnaire, surveys, interviews and ethnographic observation. Not always participation is appropriate and in some case can be risky (such as in the case of illegal topics or forms of extreme activism) or impossible (such as in the case of communities of practices). For example, in the selection of the online community under study they may use native social media devices such as mentions, like, retweets, tags, hashtags as methodological sources for selecting communities, filtering and sampling comments, posts, texts, images and videos.

Other scholars propose to use lurking as an explorative research approach. Lurking is, in this case, a useful way to explore the setting under study, learn the rules and the norm of the community, its specific organization and language. All the gained information could be used later by researchers to create interview questions (Kozinets 1998), identity gatekeepers, or choose the best strategy to introduce themselves as a newcomer to the community and get engaged in participant-observation research (Shoham 2004).

In the case of overt access, an accurate and pondered process of identity management, self-presentation strategies and researcher's interpersonal skills are fundamental. Presentation strategies can range from writing a public post in the community to explain the research goals and ask members for collaboration to try to privately contact the community administrators or key members asking them advice on how to behave within the community itself.

Whether the research is covered or uncovered, it is important to always explain the selected strategy and the reasons behind each choice. In the same way as in ethnography where ethnographers narrate the access to the field with detailed travelling and arrival stories which turn to be fundamental in making the description perceived as authentically grounded in a specific field, in netnography the access to the field needs to be narrated. The lack of physical travel does not mean that the relations between researchers and readers is totally compromised (Hine 2005). Online ethnographers can build their authority giving to the readers a detailed description of their virtual travel to the community, the used research tools, the criteria of selection, the access and negotiation strategies.

Exploring the literature (Kozinets 2002), it is possible to come across a lot of netnographic reports that provide the reader with a detailed arrival story, explaining how the researchers found the communities, why they selected them, how they negotiated access, observed members' interactions and communicated with members. These accounts do not represent an unnecessary and redundant narrative ornament, as they are fundamental for ensuring clearness, legitimizing 
the role of the netnographer, building a relationship with readers, involving them in the research process.

\section{Information Collection}

In netnographic research, data collection preserves some advantages compared with other data collection techniques such as focus group, in depth interviews and survey (see table 2). These data collection techniques are inevitably intrusive as they pose subjects in artificial and decontextualized situations. On the contrary, Netnography allows documenting the explicit language of informants without the risk of obtrusiveness and disturbance. It is focused on the study of spontaneous and naturalistic conversations, publicly available on the internet and not contaminated by the needs of a social scientist. In doing Netnography, it is not necessary to initially compile the desired data, as these data already exist in the Internet. Due to their spontaneity, the collected data are free of systematic bias, since the researcher does not interrupt the conversation and can remain invisible.

Table 3. Netnography vs. Other Research Methods: Similarities and Differences

\begin{tabular}{|l|c|c|c|c|c|c|}
\hline & Unobtrusive & $\begin{array}{c}\text { In depth } \\
\text { understanding }\end{array}$ & Naturalistic & $\begin{array}{c}\text { Less Time } \\
\text { consuming }\end{array}$ & Flexible & $\begin{array}{c}\text { Generalization } \\
\text { allowed }\end{array}$ \\
\hline Focus group & $\mathbf{X}$ & $\square$ & $\mathbf{X}$ & $\square$ & $\square$ & $\mathbf{X}$ \\
\hline Interview & $\mathbf{X}$ & $\square$ & $\mathbf{X}$ & $\square$ & $\square$ & $\mathbf{X}$ \\
\hline Survey & $\mathbf{X}$ & $\mathbf{X}$ & $\mathbf{X}$ & $\square$ & $\mathbf{X}$ & $\square$ \\
\hline $\begin{array}{l}\text { Traditional } \\
\text { ethnography }\end{array}$ & $\mathbf{X}$ & $\square$ & $\square$ & $\mathbf{X}$ & $\square$ & $\mathbf{X}$ \\
\hline Netnography & $\square$ & $\square$ & $\square$ & $\mathbf{X}$ & $\square$ & $\mathbf{X}$ \\
\hline
\end{tabular}

Source: Authors own elaboration based on Kozinets' works.

In traditional ethnography, information collection is based on different kinds of sources that can be classified in two main categories: naturally occurring and elicited (Hammersley and Atkinson 2007). The former derives from observation, participation and participants' oral accounts. The elicited are interviews, documents and artefacts of various kinds. During data collection, the researcher also has to write field notes.

As ethnography, also Netnography is multi-source: the main source of information is represented by online contents but it can be complemented by other sources such as information collected through interviews, desk analysis, etc. Exploring the literature, it is possible to come across a lot of empirical studies performed through a combination of different methods and techniques, both offline and online, in collecting and analysing data. Particularly, it is very common to come across a combination between content analysis and in depth interviews, conducted online (Maulana and Eckhardt 2014, Scaraboto and Fisher 2013, Fuller et al. 2006, Kanayama 2003); offline (La Rocca et al. 2014, Hallet and Barber 2013, Langer and Beckmann 2005) or both (Beneito-Montagut 2011). Netnography could also be combined with in person observation (Hallet and Barber 2013) and diaries analysis (Beneito-Montagut 2011, Maulana and Eckhardt 2007). It is also interesting the use of netnography within mixed method research design that 
combine online ethnography with other quantitative research methods, such as survey (Bilgram et al. 2011) or network analysis (Paccagnella 1997).

In the analysis of online communications, researchers can benefit from two main sources: the data that they directly copy from the computer-mediated interactions among community members and the field notes, i.e. data that they inscribe with their personal observations about the community, its members, interactions and meanings.

In Netnography, data collection is far less time consuming than its offline parallel. Doing an online study, in fact, the researcher can benefit from the automatic transcription of downloaded documents that are often very rich and easy to obtain.

A problem can derive, on the opposite, from the excessive availability of information, as one of most important issues of online settings is the information overload. For this reason, the researchers' choice of what is important and what is worth to consider and save should always be guided by the research aim and objectives. In this regard, Kozinets $(2002,2010)$ suggests making a preliminary distinction between on-topic and off-topic messages (where on-topic means consistent and relevant to the research aim), in order to specifically focus the research efforts on the former.

As in traditional ethnography, the other important netnographic source is represented by the reflexive field notes, where researchers record their own observations and (also) personal emotions occurring during the research. The importance of writing field notes has been underlined by many researchers (Garcia et al. 2009, Kozinets 2002) who state that field notes are very important in order to contextualize data, helping the researchers to record their experiences, describe data and develop theories from observation. The importance of the field notes is already evident at the beginning of a netnographic research. Before starting data collection, in fact, researchers can already write some guidelines containing information about the research focus and questions, the keywords used to search for the community and the criteria by which they selected the group. In the starting field notes, researchers need to clarify and formalize some important decisions, for example how is planning to interact with the community or if they are going to use a qualitative software analysis.

Apart from presenting time advantages, the online setting poses also some challenges: digital artefacts are continuously editable and most of the time, we can see the final product but not the process leading to them; what remains visible of a post is the end result without edits, changes and deletions. Fortunately, some platforms offer nethnographers useful tools to fill the gap caused by the impossibility of doing a $24 \mathrm{~h}$ observation. Facebook, for example, shows which posts have been edited and which have not and presents digital traces for the ethnographer interested in following the entire processes of meaning-making, rather than just the final product (Varis 2016).

Thus, netnographers need to choose between studying products or processes: they can limit themselves to download the data en masse (Kozinets 2010) or try to always be "there", being immersed in the situation, directly observing interactions as they take place, following in real time the processes of editing and deletion of 
posts and the flow of comments in discussion forums and blogs. However, this second option is very time and resource consuming.

Another issued relating to watching what happens on the screen, is this: there are a lot of multi-functional platforms, such as Facebook, where multiple channels of communication are available (public posts, but also private chat conversations and private messages). In other words, what is observable on the screen can be misleading, or at least provide a partial image. For example, basing the analysis only on observation, a user may seem inactive or sporadic on Facebook. The same user, indeed, could be very active in sending private messages and chatting with their connections (Varis 2016).

In both cases (lurking or participating) and like in traditional ethnographic research, data collection proceeds until theoretical saturation is reached (Glaser and Strauss 1967); additional information are supposed not to lead to significant and supplementary contributions to the research objectives. From this point of view, one of the strengths of Netnography is its ability to get in contact and study in depth an online group and its members: for this reason, interesting and useful conclusions might be drawn from a relatively small number of messages, if these messages contain rich and detailed data (Kozinets 2010).

\section{Analysing Data}

In ethnography, the step of analysis is not a distinct stage of the research: it begins in the pre-fieldwork step, with the formulation and clarification of research questions, and continues and crosses the whole research process, through to the writing of the final report. As underlined before, in ethnographic studies, the research process is always iterative, as the analysis of data feeds and affects the other research steps, like research design and data collection.

Moreover, in doing ethnography online, data analysis covers the entire process of research and it is aimed at turning the entire results of observation, participation (including textual downloaded text, graphical files, screenshot, online interviews transcripts and reflective field notes) into an organic and consistent research report.

The process of analysis cannot ignore the existing ideas of the ethnographer and the suggestions deriving from literature about the topic. However, it is important that these prior elements do not take the form of strict prejudgements, forcing interpretation of the data into categories: the researcher, instead, has to use them as useful resources to make sense of the data, being, at the same time, always open to new and unexpected interpretations, resisting the temptation to rush to determinate conclusions.

At this stage, the concepts will not usually be well-defined elements, but they will probably take the form of "sensitizing concepts" (Blumer 1954): "Where definitive concepts provide prescriptions of what to see, sensitizing concepts merely suggest directions along which to look" (Hammersley and Atkinson 2007: 164).

There are some general qualitative data processes of analysis that can be successfully applied also to netnography (Kozinets 2010, Miles and Huberman 1994): 
- coding: classifying data gathered from posts, field notes, interviews and documents into categories. During coding, names and labels are assigned to particular units of data. Categories usually emerge inductively from data themselves rather than being imposed by an a priori classification;

- noting: writing reflections and comments about the data during the collection;

- abstracting and comparing: classifying patterns into higher-order concepts, constructs or processes, identifying similar phrases, build relationships, emphasizing differences across the information;

- refinement: returning to the online community for another round of data collection in order to refine the interpretation and understanding of constructs;

- generalizing: elaborating a small set of generalizations explaining our constructs;

- theorizing: connecting our interpretation to existing theories or constructing new theories.

Apart from coding, the netnographic analysis is mostly based on a hermeneutic approach. In this regard, the concept of hermeneutic circle can be very useful. The hermeneutic circle can be considered as a methodological process for interpreting qualitative data, in which a part of qualitative data is interpreted and reinterpreted in relation to the developing sense of the whole text. According to this approach, a holistic understanding must be developed over time: initial understanding of the text is often modified as further readings provide a more developed sense of the text as a whole (Thompson 1997: 433). Doing a hermeneutic analysis, researcher needs to seek interpretations that are coherent and free of contradictions, comprehensible to the reading audience, supported with relevant examples, written in a prose that is persuasive and stimulating, using allusions, metaphors, analogies.

The two different processes of netnographic analysis, coding and hermeneutic interpretations, can be usefully combined during the analysis.

During this process, qualitative software such as NVivo or Atlas.ti could be surely useful for researchers. In particular, Paccagnella (1997) recognizes some useful tasks that qualitative analysis software could excellently perform:

- writing or transcribing field notes;

- editing, correcting, extending, or revising field notes;

- coding, i.e. attaching keywords or tags to segments of text to permit later retrieval;

- organizing storage, i.e. keeping text in an organized database;

- data linking, i.e. connecting relevant data segments to each other, forming categories, clusters, or networks of information;

- doing a quantitative content analysis: counting frequencies, sequences, or locations of words and phrases;

- conclusion-drawing and verification: aiding the analyst to interpret displayed data and to test or confirm findings;

- theory-building: developing systematic, conceptually coherent explanations of findings; testing hypotheses;

- graphic mapping: creating diagrams that depict finding or theories;

- preparing interim and final reports.

However, despite the importance of data classification and coding, Kozinets $(2002,2010)$ underlines that the most useful interpretations come as a result of a 
hermeneutic and symbolic interpretation, rather than from a meticulous process of classification. In addition, Paccagnella (1997) recognizes that, despite the different and useful tasks that a computer program could implement, it would play a supporting role for the researcher, but no computer program will analyse the data in place of the researcher. A computer program can surely make data analysis much easier, but the aim of an ethnographic research remains the study and interpretation of social phenomena provided by the researcher own hermeneutic understanding.

One of the most common data analysis practice consists in collecting data through an observation approach and analysing it by performing a bottom-up approach. In this type of study, the posts are read several times and, according to the constant comparative method (Glaser and Strauss 1967), the collected data are analysed moving from the specific to the general, identifying categories and relations between them. Even in the case of a content analysis based approach, it is possible to identity variations, as the content approach can be more focused on a qualitative interpretative approach (Hewer and Browmlie 2007, Fuller et al. 2006, Nelson and Otnes 2005) or a quantitative frequency analysis (La Rocca et al. 2014) or a combination of both Horster and Gottschalk 2012, Beaven and Laws 2007, Langer and Beckmann 2005).

\section{Netnography in Action}

Netnography is a very flexible and crosscutting research method, and it can be applied to analyse a wide and heterogeneous range of sociocultural phenomena from different disciplinary fields. Here we briefly present some examples of netnographic empirical studies conducted by our research group. For each study, a short description of research objectives and main results is provided.

\section{The Fansubbing Phenomenon}

The purpose of this research (Addeo and Esposito 2016, 2015, 2013) was to study the Fansubbing online activity, as a paradigmatic example of participatory cultures based on collaborative problem-solving and knowledge creation between people which by re-elaborating medial pre-existing materials, produce new creative forms. Fansubbing is defined as the practice of translating and creating subtitles for TV series produced in a foreign Country, usually USA or England, in order to preserve the media product in its original form, avoiding cross-cultural contaminations caused by the official dubbing. Once produced by Community members through a collective and interactive production process, subtitles are online and available to everybody. The research adopted an Exploratory Case Study approach (Yin 1994) that: "combined qualitative and quantitative techniques in data collection and analysis, following a mixed method approach. Specifically, content analysis of official documents produced by the ItalianSubsAddicted Community; press review of articles and videos about the Community; netnography for the analysis of the Community forum; in-depth interviews to 15 
Itasa members, selected with a snowball sampling" (Addeo and Esposito 2016: 23 ). The Netnography was carried out to analyse the online interactions on the most important Italian Fansubbing Community, named "ItalianSubsAddicted" (aka ITASA) which presents the highest number of users and of Series subtitled. As usual in the netnographic and ethnographic approach, research started with a foreshadowed problem ("know more about fansubbing") that turned out to be a simple start for a multifaceted and stimulating research path. After a short lurking stage, Researchers gained access to the field adopting an overt approach and collected information through a participant observation that lasted 24 months. Data analysis mixed coding and hermeneutics techniques and implemented the constant comparison method (Glaser and Strauss 1967) in which data analysis and data collection are conceived as simultaneous and interrelated processes. This progressive and iterative procedure implied that all the data already codified were constantly compared with all the new results that emerges from the analysis of new data, highlighting similarities and differences among them. Results shed light on the complex and deep learning and identity-formation dynamics of the Community, whose members group together not only to share their passion, but also to become active producer of subtitles for media products. The ITASA Community represented an interesting research subject under many points of view: "First of all, it can be considered an application of the collective intelligence dynamics: people who share the same interests and passions cooperate to make available the product of their intelligence to other people" (Addeo and Esposito 2016: 5). This results are consistent with the collective intelligence paradigm (Lévy 1994), because every Community member contributes to the group project with a distinct and specific role. ITASA could also be considered a paradigmatic example of a community of practice that allow collective and distributed learning being an effective field of learning by doing (Wenger 1998, Lave and Wenger 1991): "the Community experience, in fact, favours the development of specific skills and abilities, like linguistic skills and interpersonal skills, making the individual more competitive on the labor market" (Addeo and Esposito 2016: 5). Finally, the ITASA experience helped community members to enrich their personal culture by offering them the possibility to cooperate, and share value and opinions with different people from a wide range of social, cultural and economic conditions: "Cultural diversity tested through the Community experience allows each person to leave parochialism behind and get a new and richer identity" (ibidem).

\section{Fanfiction and Fandom}

This research shares the same ground of the previous one, as it studies an online community, Erika Fan Page (aka EFP), representing another paradigmatic example of collective intelligence. EFP was founded in 2001 by Erika, who is still managing the community as administrator. At the end of July 2019, the community has 562,559 users, 199,633 of which are registered as authors. EFP is a touchstone for fanfiction lovers and producers. In fact, this online community is dedicated to the production of fanfiction, that is the practice of re-elaborating 
media texts (TV series, Movies, Comics, Novels, and so on) in order to create new content related to the original text. For example, a fan of a specific movie can write a novel with an alternate ending of the story, or a comic fan can publish a in which. EFP community members can read and review the documents made by other members, or they can upload their own work. Under this perspective, EFP is a surely a community of practices because it creates new knowledge, by updating the existing one. EFP was also chosen because it fulfils all the selection criteria required by netnographic literature (Kozinets 2010); i.e., the EFP is relevant, active, interactive, heterogeneous, and rich of detailed and descriptive data. The access to the field was gained though several steps. First, a lurking activity was implemented in order to familiarise with the community's values, norms, roles and dynamics: "lurking phase was also useful to learn the language and the specialized vocabulary used by EFP users; without knowing it would have been impossible to understand the productive and interactive dynamics of the community" (Salzano et al. 2017: 216). After the lurking stage, researchers decided to switch to an overt access, using a mixed method approach to collect information: non-participant observation of the community members' interactions and in-depth interviews to 15 people, selected among the most active EFP users. Data collection ended when saturation was reached (Bertaux 1981, Glaser and Strauss 1987), i.e. when the researchers achieved awareness that the data collection would not have provided new insights about the research objects. Research empirical basis was made up of: online interactions among EFP users; interviews transcription; field notes. This empirical basis constantly and progressively underwent to coding, interpretation, abstraction and comparison (Silverman, 2010), thus following a grounded approach: data analysis was performed during the data collection stage, and not at the end of it. Moreover, following the aforementioned constant comparative method (Glaser and Strauss 1987), data have been analysed recursively according to the circular principle of the interpretative process (Montesperelli 1998); if new categories of analysis emerged during the collection, the already encoded information is interpreted according to the new emerging categories.

Results show that the communicative and relational practices contribute to shape EFP as a learning community in which users are able to progressively acquire and develop competences and skills related to writing and media literacy; "the dynamics which have been observed are: Fan Fiction writing as a collective autobiography and Fan Fiction communities as a digital media literacy playground" (Salzano et al. 2017: 224). Results also confirm that online communities like EFP could be seen as a social space promoting forms of engagement and peer-review practices, being so an excellent environment to develop and improve the digital media literacy. This research confirmed also that "online communities aggregating young people are interesting immersive learning environments, in which it is possible to observe the emerging of skills and competences which are not only useful within the group in terms of the construction of a reputation but also in the job market" (Salzano et al. 2017). 


\section{Gay-friendly Advertising}

This research was aimed to detect the identification factors influencing LGBT perceptions and reactions to gay-friendly advertising (Addeo et al., 2019). In this case Netnography was chosen on the basis of the unit of analysis - LGBTs. The LGBT community is probably one of the social groups that have benefitted the most from the participatory culture of the Internet. By surfing online, gay people can create or join communities in which they can easily overcome the offline difficulties of finding information, sharing experiences, feelings and interests. The social aggregation dynamics of the Web can also lead to the development of strong and influential online communities, whose members can influence the behaviour of people in the real world. For this particular community internet may serve as a socialization medium and a tool to express freely their own opinion within the gay community.

The research was based on a netnographic approach applied to the two most important Italian LGBT online communities: Gay-Forum.it and Liberamente Lesbica. These communities represent important points of reference for the Italian LBGT community and fully meet the criteria suggested by the literature on netnography (Kozinets 2010: 89): i.e. they contain relevant information related to the research focus and questions; they display recent and regular communications; they present large interactivity among participants.

In both communities, the access was preceded by a period of lurking, which is a useful covert observation for researchers to learn the norms of the groups under study, to become familiar with their organisation and to identify the community gatekeepers and key members. Then, the researchers asked the community gatekeepers for permission to start data collection and, having received the consent, they disclosed their presence to the members, presenting themselves, the aim of the study and its methodology and also contributed by co-creating contents, interacting with the community members, asking questions and giving clarifications. Data analysis was performed through a combination of coding and hermeneutic interpretation (Kozinets 2002, 2010).

Through participant observation of users' online conversations about LGBT marketing, the research highlighted the most important factors that make a brand be perceived as gay-friendly.

Overall, LGBT responses to advertising seem to vary on the basis of two dimensions which influence their identity as LGBT, the importance that they place on gay-oriented activities and their identification in corporate values:

1. the way gayness is depicted, either with stereotyped representations or with normalized representations;

2. the way sexuality is represented, either emphasising erotic connections or family values.

Stereotypical representations such as those stressing physical characteristics or attitudes (e.g. obsession with beauty and fashion, enjoying sunbathing, bars, dance and attention, assuming effeminate pose, dressed as women, etc.) are considered negative for the LGBT community because based on the difference 
with the dominant heterosexual group and on heterocentric mediatypes. Also the representation of Lesbian and Gay sexuality as an erotic lifestyle that evolve around erotic and casual sex is seen as unrealistic, depersonalising gay people as moral outsiders. Instead, LGTB community identifies in normalised alternative sexual relationships and family structures. As a consequence, the higher level of identification emerges from gay-friendly communication offering a normalized depiction of gayness not including negative stereotypes and from a representation of sexuality representing the possibility of alternative sexual relations and family structures.

\section{Airbnb Experience}

This netnographic study (Delli Paoli and Ciasullo 2019) was aimed to shed light on the symbolic meanings driving the use of shared process of consumption. Airbnb was chosen as the context of the study as it represents a good example of sharing economy based on sharing underused services directly from individuals: apartment owners (hosts) and temporary rental seekers (guests). Netnography was used as the methodology in this study as users of Airbnb are mostly active in cyberspace. Three months were spent mapping Airbnb online spaces before starting data collection in order to select the appropriate spaces for analysing online interactions between guests and hosts. The Airbnb official forum was found suitable on a few grounds. Firstly, it contains an array of relevant usergenerated information on Airbnb matters by hosts serving as a platform to gather ideas from hosts to submit to the platform and to share ideas, feedback and experience among hosts. Secondly, it is an active and interactive forum with participation from hosts from around the world following a peer-to-peer interaction model which includes also participation from Airbnb. Thirdly, with no character limit placed on the length of posts and comments, the forum is data rich. A set of selection criteria were necessary to filter through large amounts of data which were then manually stored. In order to select message threads, we used the technical features of the platform following a purposeful sampling (Patton 1990):

- Hosts' comments from the forum were downloaded and saved when they were found to be relevant in the community (they have received approval from other hosts in terms of positive votes and likes from other hosts), they have received feedback from both hosts and the platform in terms of comments by other hosts and responses from Airbnb, they included the mention of any particular meanings attributed to Airbnb experience. A total of 433 comments were analysed.

- Data from guests' reviews were downloaded and saved when they were found to be relative to hosts with listings in European capitals (London, Rome, Bruxelles, Amsterdam, Madrid, Paris, Berlin, Lisbon, Prague) with a hosting story (a high number of reviews: only the reviews with a minimum of 100 reviews were chosen to avoid one-time users) and responsive and that include reference to the specific hosting experience. Moreover, we balanced between superhosts and not superhosts, home, room and experience listings.

The study implemented a qualitative content analysis building on the 
principles of Grounded theory (Glaser and Strauss 1967) and hermeneutics (Kozinets 2010: 120). The analysis of netnographic data was guided by an inductive approach following a bottom-up approach in generating themes. It was able to identity an institutionalized system of meanings for both guests and hosts. The guests process of consumption emerged as a socially constructed and culturally embedded phenomenon which refers to experience sharing and triggers a multiplicity of emotional, physical and cognitive reactions to the guest experience involving them emotionally, physically, intellectually and spiritually. Guests are driven by the ability to have an authentic and possibly life-enriching experience that cannot be offered by competing accommodation alternatives. The hosts process of offering an accommodation is conceived as a social practice routed in the ancient traditions of hospitality and welcoming strangers. Hospitality exchange is not just considered a matter of accommodation but also a matter of meeting people and engaging in mutually meaningful social interactions. In the hosts' perspective, the concept of hospitality moves away from the businessoriented mind-set to shift toward social interaction with guests.

\section{Conclusions}

The aim of this paper was to provide an overview of Netnography as a research method. Netnography is a readily adaptable research method applicable across a wide spectrum of involvement, from lurking to active participation in online conversations and activities. Being based on creativity and bricolage, every netnographic study has distinctive and specific features that make it unique and different from other studies; for this reason, two netnographies cannot be conducted in exactly the same manner (Kozinets 2010). The methodological approaches described here are not particularly novel in themselves. They do not provide radical changes but minor adjustments to adapt traditional research methods to the specificities of new information and communication technologies. Compared with offline qualitative methods, Netnography does not provide radical shifts in practice or in epistemological substance. While imitative of traditional qualitative research, Netnography exhibits fundamental adaptations of aspects of traditional qualitative research. In Netnography, research methods need to be adapted to the contingencies of the online cultural environments: the ipertexuality of the data and the disembodied nature of online interactions. Moreover, it requires digital and hermeneutic competencies. Researchers are required to immerse themselves into the world they intend to study, to be familiar with the mechanisms of online conversations, to be able to navigate a big mass of unstructured information, interpret those assigning meanings to them and detecting the cultural connections that link content together.

In order to use Netnography researchers need to equip themselves with a natively digital methodological array using internet and online social spaces as a source of methods instead that a mere object of analysis (Caliandro 2018, Rogers 2010).

The analysis of previous netnographic research has demonstrated that: 
- netnography is particularly appropriate to those online spaces and activities that would not exist without the internet or are digitally alimented: it is the case of digital embedded practices such as in the case of fansubbing and fanfiction and online sharing practices and forms of collaborative consumption such as in the case of Airbnb.

- The definition of the online social formation under study is not necessarily the starting point of the analysis. Rather, it can be the point of arrival as in the case of Airbnb where the community was selected in different online contexts after a process of mapping users, comments, opinions, reviews using the social media logic (like, hashtag, rating, and so on).

One of the main advantage of netnography is the opportunity to expand the geographical dimension of the research field, connect dispersed networks around the world and increase the researcher's accessibility to the research population. This is particularly useful for difficult to reach groups and sensitive research topic such as illegal acts, illnesses, health concerns and interests, stigmatic phenomena and groups (migrants, disabled, LGBTs, etc.). This characteristic of netnography of being adaptable to study phenomena difficult to study through face-to-face encounters is defined by Kozinets as the "voyeuristic quality" of Netnography (Kozinets 2015: 88).

As the examples of netnographic studies presented in this paper demonstrate, the best application of netnography is to understand the social world and cultures of online communities which develop prevalently in cyberspace in order to document the explicit language of informants without the risk of obtrusiveness and disturbance of direct research involvement providing researcher with the emic (insider) and ethical (outsider) perspectives for more holistic insights. Netnography provides a valuable tool for understanding constructed online social spaces.

\section{References}

Addeo F, Esposito M (2016) Informal learning and identity formation: a case study of an Italian virtual community. In Theoretical and Applied in Psychology SICAP23. Pianoro, Bologna: 20-21 November 2015; pp. 1-6. Timisoara, Romania: Medimond International Proceedings Publisher.

Addeo F, Esposito M (2015) Processi identitari e percorsi professionalizzanti nelle comunità di pratica: il caso Itasa. In Turning around the Self. Narrazioni identitarie nel social web, D Salzano (ed), 173-179. Milano: FrancoAngeli.

Addeo F, Esposito M (2013) Collective Intelligence in Action: a Case Study Of An Italian Fansubbing Community. In ISLC-University of Izmir, Izmir, 17-10 June 2013, pp. 2091-2103 Book of Proceedings, International Symposium on Language and Communication: Exploring Novelties.

Androutsopoulos J (2006) Introduction: Sociolinguistics and computer-mediated communication. Journal of Sociolinguistics 10(4): 419-438.

Beaulieu A (2004) Mediating ethnography: objectivity and the making of ethnographies of the internet. Social Epistemology 18(2-3): 139-163.

Beaven Z, Laws C (2007) 'Never Let Me Down Again'1: Loyal customer attitudes towards ticket distribution channels for live music events: a netnographic exploration of the US leg of the Depeche Mode 2005-2006 World Tour. Managing Leisure 12(2-3): 
120-142.

Bell D (2001) An introduction to cyberculture. New York: Routledge.

Beneito-Montagut R (2011) Ethnography goes online: Towards a user-centred methodology to research interpersonal communication on the internet. Qualitative Research 11(6): 716-735.

Bertaux D (1981) From the life-history approach to the transformation of sociological practice. In Biography and society: The life history approach in the social sciences, D Bertaux (ed), 29-45. London: Sage.

Bilgram V, Bartl M, Biel S (2011) Getting closer to the consumer- How Nivea co-creates new product. Marketing Review St. Gallen 28(1): 34-40.

Blumer H (1954) What is wrong with social theory?. American Sociological Review 18: 310.

Boyd DM (2008) Taken out of context: American teen sociality in networked publics. $\mathrm{Ph}$.D. dissertation. Advisor: AnnaLee Saxenian. University of California, Berkeley.

Burawoy M, Burton A, Ferguson A, Fox KJ, Gamson J, Gartrell N (1991) Ethnography unbound: power and resistance in the modern metropolis. Berkeley, CA: University of California Press.

Burrell J (2009) The field site as a network: A strategy for locating ethnographic research. Field Methods 21(2): 181-199.

Caliandro A (2018) Digital Methods for Ethnography: analytical concepts for ethnographers exploring social media environments. Journal of contemporary ethnography 47(5): 551-578.

Campbell A (2006) The Search for Authenticity: An Exploration of an Online Skinhead Newsgroup. New Media \& Society 8(2): 269-294.

Carter D (2005) Living in Virtual Communities: An Ethnography of Human Relationships in Cyberspace. Information, Communication \& Society 8(2): 148-167.

Costello L, McDermott M-L, Wallace R (2017) Netnography: Range of Practices, Misperceptions, and Missed Opportunities. International Journal of Qualitative Methods 16: 1-12.

Cova B (1997) Community and Consumption: Towards a Definition of the "Linking Value" of Product or Services. European Journal of Marketing 31(3/4): 300-301.

Creswell JW (2008) Research Design. Qualitative, quantitative and mixed methods approaches. Thousand Oaks, CA: Sage Publications.

Delli Paoli A, Ciasullo MV (2019) The Cultural Context of Value Co-Creation. A Netnographic Study of Airbnb. [under review].

Evans M, Wedande G, Ralston L, Van Hul S (2001) Consumer interaction in the virtual area: some qualitative insights. Qualitative Market Research 4(3): 150-9.

Füller J, Jawecki G, Mühlbacher H (2006) Equipment-related knowledge creation in innovative online basketball communities. In The Future of Knowledge Management, B Renzl, K Matzler, HH Hinterhuber (eds), 161-183. Palgrave, Houndmills, Basingstoke.

Garcia AC, Standlee AI, Bechkoff J, Cui Y (2009) Ethnographic approaches to the internet and computer-mediated communication. Journal of Contemporary Ethnography 38(1): 52-84.

Geertz C (1973) The interpretation of cultures: Selected essays, vol. 5019. New York: Basic books.

Glaser BS, Strauss A (1967) The discovery of grounded theory. Chicago: Aldine.

Gossett LM, Kilker J (2006) My Job Sucks: Examining Counteristitutional Websites as Locations for Organizational Member Voice, Dissent, and Resistance. Management Communication Quarterly 20(1): 63-90.

Hallett RE, Barber K (2013) Ethnographic Research in a Cyber Era. Journal of 
Contemporary Ethnography 43(3): 306-330.

Hammersley M, Atkinson P (2007) Ethnography: Principles in practice. London: Routledge.

Heath D, Koch E, Ley B, Montoya M (1999) Nodes and queries: Linking locations in networked fields of inquiry. The American Behavioural Scientist 43: 450-63.

Hewer P, Brownlie D (2007) Cultures of consumption of car aficionados: Aesthetics and consumption communities. International Journal of Sociology and Social Policy 27(3/4): 106-119.

Hine C (2005) Virtual methods. New York: Berg Publishers.

Hjorth L, Horst H, Galloway A, Bell G (eds) (2017) The Routledge Companion to Digital Ethnography. Taylor \& Francis.

Horster E, Gottschalk C (2012) Computer-assisted Webnography A new approach to online reputation management in tourism. Journal of Vacation Marketing 18(3): 229238.

Kanayama T (2003) Ethnographic research on the experience of Japanese elderly people online. New Media \& Society 5(2): 267-288.

Kozinets RV (1998) On Netnography: Initial reflections on consumer research investigations of cyberculture. Advances in Consumer Research 25(1): 366-371.

Kozinets RV (2002) The Field Behind the Screen. Journal of Marketing Research 39(1): 61-72.

Kozinets RV (2010) Netnography: Doing ethnographic research online. London: Sage Publications.

Kozinets RV (2015) Netnography: Redefined. London, England: Sage.

La Rocca A, Mandelli A, Snehota I (2014) Netnography approach as a tool for marketing research: the case of Dash-P\&G/TTV. Management Decision 52(4): 689-704.

Langer R, Beckman SC (2005) Sensitive research topics: Netnography revisited. Qualitative Market Research: An International Journal 8(2): 189-203.

Lave J, Wenger E (1991) Situated Learning: Legitimate Peripheral Participation. Cambridge: Cambridge University Press.

Lévy P (1994) L'Intelligence collective. Pour une anthropologie du cyberespace. Paris: La Découverte.

Maclaran P, Catterall M (2002) Researching the social web: marketing information from virtual communities. Marketing Intelligence \& Planning 20(6): 319-326.

Madge C, O'Connor H (2006) Parenting Gone Wired: Empowering of New Mothers on the Internet? Social \& Cultural Geography 7(2): 199-220.

Maffesoli M (1996) The Time of Tribes: The Decline of Individualism in Mass Society. London: Sage Publications.

Malinowski B (2002) Argonauts of the Western Pacific: An account of native enterprise and adventure in the archipelagoes of Melanesian New Guinea. London: Routledge.

Mann C, Stewart F (2000) Internet communication and qualitative research: A handbook for researching online. London: Sage Publications.

Marradi A (2007) Metodologia delle Scienze Sociali. Bologna: Il Mulino.

Marzano M (2006) Etnografia e ricerca sociale. Bari: Laterza.

Maulana AE, Eckhardt GM (2007) Just friends, good acquaintances or soul mates? An exploration of web site connectedness. Qualitative Market Research: An International Journal 10(3): 227-242.

Miles MB, Huberman AM (1994) Qualitative data analysis: An expanded sourcebook. London: Sage Publications.

Montesperelli P (1998) L'intervista ermeneutica. Milano: FrancoAngeli.

Morton H (2001) Computer-mediated communication in Australian anthropology and sociology. Social Analysis 45(1): 3-11. 
Murthy D (2008) Digital ethnography an examination of the use of new technologies for social research. Sociology 42(5): 837-855.

Nelson MR, Otnes C (2005) Exploring cross-cultural ambivalence: A Netnography of intercultural wedding message boards. Journal of Business Research 58(1): 89-95.

Olaniran B (2008) Electronic Tribes (E-Tribes): Some Theoretical Perspectives and Implications. In Electronic Tribes: Virtual Worlds of Geeks, Gamers, Shamans, and Scammers, TL Adams, SA Smith (eds), 36-57. Austin, TX: University of Texas Press.

Paccagnella L (1997) Getting the seats of your pants dirty: Strategies for ethnographic research on virtual communities. Journal of Computer-Mediated Communication $3(1)$.

Patton MQ (1990) Qualitative evaluation and research methods. Thousand Oaks, CA: Sage.

Postill J, Pink S (2012) Social media ethnography: the digital researcher in a messy web. Media International Australia 145: 123-134.

Puri A (2007) The web of insight. The Art and Practice of Webnography. International Journal of Market Research 49(3): 387-408.

Rheingold H (1993) The virtual community: Homesteading on the electronic frontier. Reading, Massachusetts: MIT press.

Riffe D, Lacy S, Fico F, Watson B (2019) Analyzing Media Messages. Using Quantitative Content Analysis in Research, $4^{\text {th }}$ ed. New York: Routledge.

Robinson L, Schulz J (2011) New field sites, new methods: new ethnographic opportunities. In The Handbook of Emergent Technologies in Social Research, 180198. Oxford: Oxford University Press.

Rogers R (2010) Internet research: the question of method: a keynote address from the YouTube and the 2008 Election cycle in the United States conference. Journal of Information Technology \& Politics 7: 241-60.

Rogers R (2013) Digital methods. Cambridge MA: MIT Press.

Salzano D, Addeo F, Napoli A, Esposito M (2017) Paideia Between Online and Offline: A Netnographic Research on Fan Fiction Communities. Italian Journal of Sociology of Education 9(3): 207-235.

Scaraboto D, Fischer E (2013) Frustrated fatshionistas: an institutional theory perspective on consumer quests for greater choice in mainstream markets, Journal of Consumer Research 39(6): 1234-1257.

Shoham A (2004) Flow experiences and image making: An online chat-room ethnography. Psychology \& Marketing 21(10): 855.

Silverman D (eds) (2010) Qualitative research. London: Sage.

Thompson CJ (1997) Interpreting Consumers: A Hermeneutical Framework for Deriving Marketing Insights from the Texts of Consumers' Consumption Stories. Journal of Consumer Research 34 (November): 438-55.

Tuncalp D, Lé PL (2014) (Re)Locating boundaries: a systematic review of online ethnography. Journal of Organizational Ethnography 3(1): 59-79.

Varis P (2016) Digital ethnography. In The Routledge Handbook of Language and Digital Communication, A Georgakopoulou, T Spilioti (eds), 55-68. London: Routledge.

Wenger E (1998) Communities of practice: Learning, meaning, and identity. Cambridge: Cambridge University Press.

Williams JP and Copes H (2005) How Edge are you? Constructing Authentic Identities and Subcultural Boundaries in a Straightedge Internet Forum. Symbolic Interaction 28(1): 67-89.

Yin R. (1994) Case study research: Design and methods, $2^{\text {nd }}$ ed. Thousand Oaks, CA: Sage Publishing. 
